\title{
Nuestro mundo cubierto de plástico: de la movilidad global del plástico a las consecuencias y respuestas locales
}

\section{Our world covered in plastic: from the global mobility of plastic to local consequences and responses}

\author{
Marisol Espino Penilla, Yenthl Koot \\ marisol.espinopenilla@wur.nl \\ Wageningen University and Research, Países Bajos
}

Recibido: 28/05/2020. Aceptado: 14/12/2020

\section{RESUMEN}

La contaminación plástica es un problema mundial importante en la realidad actual, causado por los patrones de producción, consumo y pos-consumo de plástico. Los plásticos se han globalizado y el turismo contribuye a este proceso. Este documento busca desentrañar los diferentes impulsores detrás de la movilidad global del plástico y analizar cómo el turismo contribuye y se ve afectado por este proceso. Examina la naturaleza del problema de los residuos plásticos y cómo se intensifica con los efectos globalizadores de la industria del turismo. El caso de la Isla de Pascua, que se encuentra cerca del parche de basura más grande en el Gran Océano Pacífico, se utiliza para ilustrar cómo la movilidad global de los flujos de plástico afecta a las localidades en islas remotas cuyas economías dependen del turismo. Este caso nos permite hacer una evaluación minuciosa de la escala global de la contaminación plástica.

Palabras clave: contaminación plástica; turismo; islas remotas; movilidad global de plásticos; Isla de Pascua; Parche de Basura del Pacífico.

\begin{abstract}
Plastic pollution is an important global problem in today's reality, caused by the patterns of production, consumption and post-consumption of plastic. Plastics have become globalized and tourism contributes to this process. This paper seeks to unravel the different drivers behind the global mobility of plastic and analyze how tourism contributes to and is affected by this process. It examines the nature of the plastic waste problem and how it is intensified by the globalizing effects of the tourism industry. The case of Easter Island, which is located near the largest garbage patch in the Greater Pacific Ocean, is used to illustrate how the global mobility of plastic flows affects localities on remote islands whose economies depend on tourism. This case allows us to make a thorough assessment of the global scale of plastic pollution.
\end{abstract}

Keywords: plastic pollution; tourism; remote islands; global plastics mobility; Easter Island; Pacific Garbage Patch. 


\section{INTRODUCCIÓN}

La "globalización" es uno de los conceptos de moda de nuestro tiempo, lo escuchamos cada vez más en las noticias, como parte de los discursos políticos y científicos, y se ha integrado altamente en las estrategias comerciales (Munar, A.M., 2007). Los aumentos drásticos en los flujos de información, capital, inversión extranjera, materiales y comercio transnacional derivan en la necesidad de comprender las razones y las maneras en las que sucede. El concepto de globalización ha sido propuesto para comprender estos cambios y fue definido por Al-Rodhan y Stoudmann (2006, p. 2) como un "proceso que abarca las causas, el curso y las consecuencias de la integración transnacional y transcultural de actividades humanas y no humanas". Por otro lado, se ha descubierto que el comercio internacional como parte del proceso de globalización tiene efectos contradictorios sobre el medio ambiente, al mismo tiempo aumentando los niveles de contaminación y motivando su mitigación. Estos efectos están distribuidos de manera diferenciada alrededor del mundo, resultando en la disminución de los niveles de contaminación de los países desarrollados, y en un aumento en los países en desarrollo, este último siendo el efecto mayor (Dinda, S., 2006).

Sin embargo, podríamos argumentar que la contaminación es en sí misma un problema global. En 2015, se generaron alrededor de 1.300 millones de toneladas de residuos sólidos urbanos en todo el mundo y se espera se duplique para el 2025, impulsado por la urbanización y el desarrollo socioeconómico en los países en desarrollo (Hoornweg y Bhada-Tata, 2012, según lo citado en Turner et al., 2015). A su vez, los residuos se relacionan con el cambio climático en una escala mundial. Existe una doble contribución de los residuos al cambio climático, ya que la disposición final de materiales en los vertederos contribuye no solo a través de las emisiones de gases de efecto invernadero que se producen en este proceso, sino que también se descartan recursos útiles, eliminando la posibilidad de reinsertarlos en el ciclo de producción, por lo tanto, representan la necesidad de una nueva extracción, procesamiento, transporte y uso de recursos naturales "vírgenes" (Turner et al., 2015).

Los términos "residuos" y "contaminación" abarcan muchos materiales y procesos diferentes, por lo tanto, en este artículo, nos enfocaremos en los plásticos como un ejemplo de residuos globalizados. Nos referimos a los plásticos como globalizados porque son altamente comercializados internacionalmente, especialmente porque se usan como envases para una amplia gama de productos de comercio tanto nacional como internacional. El plástico es extremadamente móvil en todas las etapas de su vida. El plástico es altamente móvil desde su producción, en su vida útil como producto o envase, e inclusive cuando se vuelve residuo. Los movimientos de los plásticos pueden entenderse mejor como flujos. Estos flujos y movimientos no son únicamente inducidos por actividades humanas, sino también suceden cuando los plásticos entran en contacto con vientos y corrientes oceánicas. Los procesos naturales que capturan estos materiales y causan su movimiento, lo hacen sin tener en cuenta las fronteras nacionales. En el contexto de movilidad global de los plásticos, los factores económicos y los procesos naturales que distribuyen la contaminación plástica y sus efectos negativos lo hacen sin respetar los conceptos humanos de equidad y responsabilidad.

La cantidad de plásticos producidos en el mundo es alarmante, los investigadores han descubierto que el plástico producido entre 1950 y 2015 es "más que suficiente para envolver todo el planeta en una capa de film transparente" (Gross, 2017, p. 785). El plástico ejemplifica la doble contribución de los residuos en el cambio climático, ya que su proceso de extracción, producción y transporte conlleva uso de combustibles y la mayor cantidad de los plásticos producidos en la historia no se han reciclado, sino que se han quemado, enterrado en rellenos 
sanitarios o desechado de manera inapropiada, lo que la mayoría del tiempo significa que termina en el océano.

Los residuos plásticos que terminan en los océanos son una de las principales preocupaciones ambientales de nuestros tiempos y las islas remotas se ven particularmente afectadas por grandes cantidades de residuos plásticos que terminan en sus costas. Aunque la lejanía alguna vez fue una garantía de protección contra la contaminación plástica, el plástico ahora está llegando a estas islas a través de las corrientes oceánicas y el viento. Según Gross (2017), "Las islas remotas simplemente actúan como un sumidero, filtrando parte de la basura de los océanos y permitiendo a los científicos una evaluación detallada de la escala del problema" (p.785). Estas islas pueden verse como depósitos temporales en los que se acumula el plástico. Además de las corrientes oceánicas y el viento, otro factor importante responsable de la cantidad de plástico en las islas remotas es el desarrollo del turismo, ya que el turismo aumenta la demanda de envases de un solo uso, especialmente para alimentos y bebidas (Monteiro et al, 2018). Las islas remotas son un ejemplo perfecto de cómo algunos destinos luchan cada vez más para hacer frente a la rápida expansión del turismo (Wood et al, 2019). Wahab y Cooper (2001) describen la expansión del turismo como un lugar de esparcimiento para las fuerzas mundiales, que incrementan debido a la creciente riqueza de muchas naciones, el aumento en la cantidad y variedad de motivaciones de viaje, la mejora de los viajes aéreos y los movimientos de desregulación o liberalización. Los habitantes locales luchan para lidiar con este problema, ya que las islas remotas a menudo tienen recursos limitados, espacio reducido y opciones de gestión de residuos insuficientes (Monteiro et al, 2018).

Junto con el aspecto geográfico de las islas remotas, el aislamiento también se define por la falta de lazos comerciales de la isla con el resto del mundo y sus impactos antropogénicos mínimos (Heskett et al, 2012). Dinda (2006) se refiere a los lazos comerciales en términos de comercio como la causa de la degradación ambiental en las islas, ya que el aumento del comercio ampliaría el tamaño de la economía de la isla y aumentaría la contaminación, lo que disminuye la calidad ambiental. En las islas donde alguna vez hubo pocos efectos negativos sobre el medio ambiente, que podrían ser resultado de la baja densidad de población, el acceso limitado a la tecnología y la falta de mercados externos (Hames, 2007), ahora hay una mayor degradación de los recursos en parte debido al desarrollo turístico y sus efectos secundarios. Esta degradación natural en las islas remotas afecta al bienestar económico de las empresas y habitantes locales ya que la condición prístina y la belleza natural se encuentran entre los principales factores de atracción turística. Los medios de sustento de la población local pueden verse afectados cuando los turistas deciden que la calidad de los recursos naturales del lugar ya no es suficiente para que valga la pena visitar.

\section{SOCIOLOGÍA DE FLUJOS AMBIENTALES PARA ENTENDER LA GLOBALIZACIÓN PLÁSTICA}

Este documento tiene como objetivo explorar la forma en que ocurrió la globalización de los flujos de plástico, y la manera en qué esta movilidad global del plástico ha influenciado y sido influenciada por el turismo, así como cuáles son las principales consecuencias de este proceso. Para hacer esto, el estudio se basa en la teoría de Mol y Spaargaren sobre la Sociología de flujos ambientales (2005) que se desarrolla en torno a cuatro temas principales: la definición de flujos, la relación entre lo social y lo material, cuestiones de poder y desigualdad, y la gobernanza global. En la Sociología de flujos ambientales, el enfoque en los flujos o movimientos de materiales es fundamental, pero a su vez, también se reconoce el 
contexto social en el que estos movimientos ocurren, haciendo los flujos de materiales inherentemente sociales. Esta combinación del mundo natural o material con el social, da como resultado lo que Urry (2003, como se indica en Mol y Spaargaren, 2005) llamo "híbridos". Es precisamente en esta intersección, en un mundo híbrido, en donde la Sociología de flujos ambientales centra su análisis. Además, como apoyo para el análisis de poder y de las desigualdades sociales, se utilizará un enfoque de ecología política con el objetivo de descubrir los poderes políticos y económicos que dominan los flujos que conducen al plástico a lugares remotos. La ecología política tiene como uno de sus enfoques centrales el conflicto ambiental y sus efectos de distribución (Alier, 1995, como se indica en Escobar, 1998). Estos aspectos son clave para este análisis, ya que examinamos las consecuencias sociales y ambientales de la distribución del plástico en lugares remotos causadas por la globalización.

En la segunda mitad del documento, el caso de la Isla de Pascua se utilizará para ilustrar cómo el turismo, los flujos de plástico, las regulaciones gubernamentales y la globalización juegan un papel en los problemas de contaminación plástica de la remota isla, y cuáles son las consecuencias y respuestas locales. Actualmente, los científicos están explorando los efectos que la globalización, el turismo y la contaminación tienen en la isla. Desde que la isla ganó más atención de los medios, los locales y científicos interesados están cada vez más preocupados por el equilibrio entre el turismo y los esfuerzos de preservación. Los habitantes de la Isla de Pascua han estado abogando por el medio ambiente durante mucho tiempo, porque sus medios de sustento dependen en gran medida de la calidad del entorno natural de la isla. La isla está batallando para solucionar los problemas que genera el plástico acumulado, los cuales se acentúan al combinarse con los recursos limitados del área (Blichert, 2019).

\section{MOVILIDAD GLOBAL DEL PLÁSTICO}

Los plásticos son conceptualizados como un flujo ambiental en este artículo debido a las extensivas cantidades de este material que se movilizan globalmente, sin estar limitados por fronteras o practicas estáticas y que a su vez están afectados por una numerosa cantidad de cuestiones sociales, como practicas económicas globales, políticas, discursos, etc.. Al hacer esto, se puede visualizar cómo Mol y Spaargaren (2005) analizan los flujos ambientales en relación con las instituciones de la sociedad moderna. El análisis consiste en observar el movimiento de los flujos mundiales de materiales entre las actividades sociales de producción y consumo y el medio ambiente natural. En las siguientes secciones desarrollaremos más esta idea, siguiendo el flujo de plástico empezando por su producción, siguiendo por el comercio hasta la eliminación y el reciclaje.

\subsection{De la producción al comercio}

El plástico es un material valioso, ya que es barato de producir, puede ahorrar recursos en comparación con otros materiales, es versátil, liviano y resistente. Actualmente es difícil imaginar un mundo sin el plástico como contribuyente clave para nuestra sociedad. Los plásticos también permiten la innovación en muchos sectores de nuestra sociedad a través del desarrollo de productos y soluciones que no existirían sin este material (PlasticsEurope, 2017). En términos de beneficios medioambientales, el plástico juega un papel importante en el mantenimiento de la calidad de los alimentos y en la prevención del desperdicio (Ritchie \& Roser, 2018). Existe entonces una disonancia en la que el plástico se utiliza como herramienta para evitar el desperdicio como recipientes de alimentos, pero se convierte en desperdicio inmediatamente después que el alimento es consumido. En la industria, se hace una distinción 
entre plásticos duraderos y no duraderos, donde los plásticos duraderos se refieren a productos que tienen un ciclo de vida de al menos tres años, como muebles, productos electrónicos y materiales de construcción, mientras que los plásticos no duraderos, como envases, botellas, bolsas, vasos y otros artículos desechables, a menudo de un solo uso, son útiles durante menos de tres años (American Chemistry Council, 2019). Los plásticos no duraderos serán nuestro enfoque por dos razones principales. Primero, el mercado más grande de plásticos a nivel mundial y el más usado en la industria del turismo, es el embalaje. En segundo lugar, estos tipos de plásticos son los que tienen un ciclo de vida del producto corto, por lo que se convierten en residuos rápidamente después de haber sido producidos.

En los años 50, la producción mundial de plástico fue de 2 millones de toneladas al año, en 2015 alcanzó los 381 millones de toneladas y continúa creciendo. China es el mayor productor de plástico, con más de una cuarta parte de la producción mundial y aumentando (Ritchie \& Roser, 2018). El segundo mayor productor es Europa, el cual produjo 60 millones de toneladas de plástico en 2016 (PlasticsEurope, 2017). Es en esta etapa se pueden observar las relaciones comerciales que se generan con el plástico entre diferentes continentes y países. El comercio es entonces identificado como el primer motor para la globalización de este material. Europa, por ejemplo, exportó el 17,6\% de sus plásticos fabricados a China e importó el 18,1\% de Arabia Saudita y el 15,8\% de Corea del Sur (PlasticsEurope, 2017). Las relaciones de poder que rigen estos flujos materiales, representadas por cuestiones económicas, son una parte esencial de la sociología de flujos (Spaargaren, 2005) y pueden ser visualizadas el tipo de plásticos (en calidad y precio) que se comercializan internacionalmente.

Sin embargo, el plástico no suele venderse a su último consumidor por sí solo. Los comercios $\mathrm{y}$ supermercados se encuentran en un punto medio entre el consumidor final y los productores. Tomando el ejemplo de un supermercado común, más de la mitad de los productos que podemos encontrar tienen algún tipo de empaque de plástico (botellas, bandejas, bolsas, etc.) que ayuda al productor a hacer que sus productos sean móviles al facilitar su transportación. La Federación Británica de Plásticos afirma que "sin los envases de plástico, muchos productos que los consumidores compran no viajarían a la casa o la tienda, ni sobrevivirían en buenas condiciones el tiempo suficiente para ser consumidos o utilizados" ("About Plastics Packaging", 2019). Este es el caso de todos los líquidos (agua, refrescos, leche, jabón, etc.), cereales y granos, alimentos prefabricados, pan, especias, carne y muchos otros comestibles y bienes de consumo de rápido movimiento. Los supermercados y otras tiendas, por lo tanto, son responsables por el comercio de una gran parte de los plásticos producidos en el mundo. El número es tal, que en lugares como el Reino Unido, se han firmado acuerdos de confidencialidad con agencias de reciclaje para mantener estos números en secreto de los medios (Laville y Taylor, 2018). Este despliegue de poder de la industria comercial sobre las entidades de reciclaje dio forma a los discursos e información disponible sobre el plástico, a su vez moldeando y direccionando los flujos del mismo.

\subsection{El problema del "reciclaje"}

En el mundo del plástico, existen algunos países que actúan como actores clave en la importación de residuos. Hasta el 2018, China había sido la más importante, ya que importaba el $46 \%$ de los residuos plásticos del mundo. La prohibición que China impuso a la importación de residuos plásticos en el 2018 fue un problema global que impactó varias localidades a nivel mundial. Europa había estado exportando el $40 \%$ de sus residuos plásticos a China durante años (Zoete, 2018). El crecimiento económico en China, el incremento del consumismo en el país y la creciente conciencia de la contaminación ambiental y la salud 
pública, hizo que China se independizara del reciclaje de los desechos de otros países y se centrara en el reciclaje de las cada vez mayores cantidades de plástico nacional. La prohibición de la importación de residuos plásticos globales en China se decidió ya que gran parte de los plásticos importados fueron almacenados y eventualmente incinerados debido a la falta de capacidad de almacenamiento y la imposibilidad de reciclar ciertos tipos de plástico (Zoete, 2018).

Después del cierre de la frontera para las importaciones de plástico en China, las exportaciones de residuos plásticos principalmente de los Estados Unidos y Europa se desviaron a Malasia, Tailandia, Vietnam y Taiwán (GAIA, 2019). Es así como una decisión nacional en China, tuvo repercusiones en varios otros países y a diferentes niveles. Al analizar el movimiento del plástico, conceptualizado como un flujo ambiental, se destacan cuestiones sobre control de movilidad, que reflejan y refuerzan estructuras de poder (Sheller \& Urry, 2006) y que se ven ejemplificadas en el proceso de reciclaje global de plásticos. Los flujos de plástico fueron desplazados de países desarrollados hacia otros destinos, la mayoría de estos ubicados en países en desarrollo y localidades de bajos recursos que no cuentan con la capacidad de procesamiento para la cantidad de plásticos que son transportados hacia ellos y que se ven gravemente afectados por su presencia.

\subsection{Movimiento de los plásticos en el océano}

Cuando los plásticos se desechan en el mar, son transportados por corrientes complejas que los transportan por todo el mundo y eventualmente los llevan a uno de los cinco "parches de basura" distintos en los océanos subtropicales (Sebille, 2015). Estas corrientes oceánicas superficiales se crean en parte por vientos que soplan sobre el océano y ayudan a crear un sistema complejo de corrientes marinas (Price, Weller y Schudlich, 1987). Estas corrientes de viento y agua cambian profundamente los flujos de plástico y le dan poder de distribución a los elementos no humanos (por ejemplo, el océano y el viento). Sebilles (2015), realizó un estudio en el que lanzaron varios derivadores oceánicos, llevando un dispositivo GPS, con no más de 13 metros entre ellos. Los resultados mostraron que, incluso cuando se liberaron a poca distancia el uno del otro, en unas pocas semanas los derivadores tenían varios cientos de kilómetros entre sí. Por impredecibles que puedan parecer estas corrientes, eventualmente la mayoría de estos flujos conducen a uno de los cinco patrones de circulación subtropical a gran escala, mejor conocidos como Gyres o "parches de basura oceánica". Sin embargo, pueden pasar varias décadas antes de que los plásticos lleguen a algunos de estos giros y la mayoría del plástico que hay actualmente en los océanos todavía está "en camino". Como estos parches generalmente coinciden con regiones del océano con bajos niveles de nutrientes, no hay mucha fauna marina a su alrededor, lo que hace que el "plástico ambulante" sea el de mayor riesgo para los organismos marinos (Sebille, 2015).

\subsection{El turismo como causa}

Los residuos plásticos en islas remotas generados como consecuencia del turismo son un ejemplo de los efectos negativos de la globalización. Los plásticos tienen varias formas de ingresar a las islas influenciados por el turismo. Muchos estudios han demostrado que el turismo contribuye al aumento de la presencia de residuos plásticos en el océano, y en zonas de turismo intensivo, la influencia es tan contundente que significa un aumento de hasta $70 \%$ (Ivar do Sul et al., 2011; Gabbatiss, 2018; Monteiro, Ivar do Sul \& Costa, 2017). Este número es impresionante, ya que solo representa el plástico adicional que termina en el océano, sin tener en cuenta la cantidad de plástico que se recicla, se entierra o se quema. Generalmente, el turismo es identificado como una actividad social de consumo y no de producción de plásticos. Sin embargo, el turismo también influye en la producción de plástico al modificar 
los patrones de consumo. Por lo tanto, argumentamos que la influencia del turismo en la movilización del plástico puede ser directa (consumo) o indirecta (producción) y que al analizar los principales impulsores detrás de los flujos de estos materiales, podemos comprender mejor el papel que la interrelación de la industria turística con la red de movilidad global del plástico tiene en este proceso de movilización.

\subsubsection{Influencias directas del turismo}

Muchos turistas transportan plásticos en sus equipajes, generando así una influencia directa y personal en los flujos globales de plástico. Sin embargo, el mayor impacto de los turistas en la generación de plásticos se da por las prácticas y expectativas de consumo que conlleva el movimiento humano. Cuando una persona viaja, no viaja únicamente su cuerpo, sino que también transporta en sí una serie de ideologías y expectativas a donde quiera que va. Es así como las influencias directas del turismo en los flujos del plástico son representadas por los plásticos generados en todas las actividades turísticas (por ejemplo, alojamiento, restaurantes, excursiones, transportes, etc.). Los diez plásticos más comunes que pueden explicarse por las actividades turísticas son empaques y envoltorios de alimentos, pajillas, tazas, bolsas, botellas de bebidas, envases para llevar, vasos (de espuma y plásticos), colillas de cigarrillos, tetrapacks y tapas (5GYRES et al. , 2016, Ivar do Sul et al., 2011). Esta lista pone de manifiesto que el principal impulsor directo de la movilización del plástico es el transporte y consumo de alimentos y bebidas y coincide con el estudio de Monteiro et al. (2017) en el que los alimentos, bebidas y envases de un solo uso se consideraron como la contribución más relevante del turismo a los residuos plásticos.

Los proveedores locales de servicios, supermercados, hoteles y restaurantes consumen y comercializan productos contenidos en plástico necesarios para cubrir las necesidades que los turistas traen consigo. De esta manera los turistas tienen un doble impacto en la generación de residuos plásticos ya que no sólo transportan el material, sino también moldean la cadena de suministro de la isla. Los plásticos que se traen con los turistas del continente a las islas remotas y que afectan a las poblaciones locales, conllevan el movimiento de estos materiales de un lugar a otro, a veces incluso a un nivel internacional, ejemplificando así la movilidad global de materiales. El movimiento del plástico está siendo forzado por la idea de que muchas islas remotas no tienen suficientes recursos para atender a todos los turistas, lo que en muchos casos es cierto. Sin embargo, lo que muchos turistas y proveedores de servicios ignoran o deciden pasar por alto, es que una vez que compran algo de plástico en un lugar y lo tiran en otro lugar que no puede reciclar la cantidad de residuos plásticos, están generando daños al destino y a su gente.

\subsubsection{Influencias indirectas del turismo}

Las influencias indirectas del turismo en la globalización del plástico se conceptualizan como aquellos movimientos de plástico generados no por el consumo directo del turista, sino como resultado de todas las actividades de soporte que se llevan a cabo para asegurarse de satisfacer las necesidades de los turistas. Dos ejemplos útiles y relevantes en islas remotas serían los efectos del turismo en la industria pesquera y los flujos de migración humana. Primero, la industria pesquera está incluida como uno de los tres principales contribuyentes a la contaminación plástica (Sheavly \& Register, 2007). La pesca y el turismo se han relacionado históricamente de varias maneras, ya que el turismo generalmente se considera una alternativa de subsistencia "sostenible" a la pesca a gran escala en las zonas costeras (Fabinyi, 2010) y, al mismo tiempo, el turismo aumenta la demanda local de pescado. El suministro de alimentos se encuentra entre las principales fuentes de ingresos en la industria turística (Divisekera, 2010). Este importante movilizador económico hace que el proporcionar pescado fresco a los 
restaurantes sea atractivo para los pescadores locales. Esto aunado a las cantidades de pesca que ya tenían que proporcionar antes del desarrollo del turismo.

Segundo, el turismo ha sido reconocido como una causa relevante de flujos migratorios (Williams y Hall, 2010), ya que genera un mercado laboral que con frecuencia no puede ser cubierto localmente, sobre todo en áreas remotas (Monk y Alexander, 1986 como se cita en Williams y Hall, 2010). Las personas que se mudan a estas áreas, generalmente tienen diferentes nacionalidades, habilidades, géneros o etnias, dependiendo de las necesidades de la industria del turismo que los locales no pueden satisfacer (King, 1995, citado en Williams \& Hall, 2010) y la mayoría tiempo, también tienen diferentes estilos de vida que los locales (Snepenger wt al., 1995, como se cita en Williams \& Hall, 2010). Estas diferencias en los estilos de vida conducen a cambios en los patrones de consumo local, ya que los estilos de vida requieren un conjunto específico de materialidades, lo que generalmente incluye un aumento en el uso de plástico.

\subsection{Influencias de los residuos plásticos en el turismo}

La relación del turismo con los residuos plásticos es compleja, ya que el turismo es por un lado responsable de su generación, pero al mismo tiempo es directamente afectada por su existencia. La acumulación y disposición incorrecta de los residuos plásticos disminuye el atractivo de un destino y también la calidad de vida sus habitantes. A la larga, esto tiene efectos negativos en la participación sostenible de la comunidad local en el turismo, ya que genera fricciones $\mathrm{y}$ percepciones negativas entre locales $\mathrm{y}$ turistas $\mathrm{y} / \mathrm{o}$ proveedores de servicios. Estos efectos negativos han sido percibidos por varios destinos, llamando así la atención de varios organismos y generando diferentes tipos de respuestas.

Por lo general, se puede llegar a las islas tanto por vía área como por agua. Estos movimientos permiten la movilidad tanto de personas como de materiales (incluidos los plásticos). Por lo tanto, restringir el uso de plástico en las islas se ocupa de "una red compleja con intersecciones de regímenes interminables de flujos que se mueven a diferentes velocidades, escalas y viscosidades" (Sheller \& Urry, 2006, p.213). Al relacionarlo con la sociología de flujos ambientales de Mol y Spaargaren (2005), podemos entender la relación entre el turismo y el plástico como parte una red que opera en un contexto global y de alta velocidad, en la que las dinámicas de poder juegan un papel destacado. Dentro de esta "red" existen algunos actores dominantes que pueden hacer cumplir o resistir fácilmente los despliegues de poder.

Un actor dominante dentro de la industria del turismo es Travelife, que es una iniciativa líder de capacitación, gestión y certificación para empresas turísticas. Junto con otras partes interesadas, Travelife para hoteles y alojamientos lanza cada año una campaña para alentar a la industria y consumidores del turismo a tomar medidas más amigables con el ambiente. En 2018, el tema fue 'Di no al plástico', y el tema de este año (2019) es 'Reducir, reutilizar, reciclar', en el que continúan enfocándose en los plásticos y desechos globales, llevándolo así a un nivel más amplio (ABTA, 2019). Grandes cadenas hoteleras como Marriott, Hilton, Hyatt y Four Seasons dijeron que habían prohibido los sorbetes, y algunas de ellas también proporcionaron alternativas para botellas de champú o de bebidas en botellas de plástico y en su lugar proporcionaron a los huéspedes unas reutilizables (Hospitality Insights, 2019).

Sin embargo, una de las razones subyacentes para que los turistas compren botellas de agua de plástico es porque el agua del grifo en muchos países no se puede beber. En lugar de proporcionar a las personas botellas reutilizables, debe hacerse algo para potabilizar el agua $\mathrm{u}$ otras formas que permitan un acceso general al agua potable limpia sin necesidad de usar plástico. Existe un creciente interés mundial en crear redes e iniciativas para proporcionar 
acceso a agua limpia a la población mundial, que también es uno de los Objetivos de Desarrollo Sostenible de la ONU. Desafortunadamente, el turismo a menudo se ve como una industria completamente diferente y las necesidades de provisión de infraestructura son puestas detrás de otros intereses (económicos).

\section{ISLA DE PASCUA: CONSECUENCIAS LOCALES Y RESPUESTAS}

Entre otras características específicas, los paisajes extraordinarios y su lejanía de la "civilización" son los mayores atractivos turísticos de la Isla de Pascua. Ritchie y Zins (1978) identifican muchos otros factores de atracción de un destino, incluida la belleza natural, el clima, y las características culturales y sociales que están muy presentes en la isla. En esta sección, analizaremos las consecuencias de la globalización, el turismo y el plástico a nivel local y presentaremos algunas de las iniciativas y respuestas de la isla.

Ubicada en el Océano Pacífico Sur, la Isla de Pascua es conocida por su lejanía, las estatuas de piedra Moai y, según el sitio web de la junta de turismo de la isla, por las aguas cristalinas con perfectas condiciones de buceo (Easter Island Tourism, 2019). Sin embargo, las aguas que rodean la isla contienen una de las mayores concentraciones de microplásticos del mundo (Hardin, 2019). Estos plásticos no llegan solo a través de las corrientes oceánicas y el viento, sino que también a través de la creciente industria turística (Hardin, 2019). Aunque el problema plástico de la Isla de Pascua no es nada nuevo, recientemente ganó la atención de los medios de comunicación mundiales. El aumento en la cantidad de contaminación plástica en la Isla de Pascua es visible desde 1997, el año en que los científicos descubrieron que el área que rodeaba la Isla de Pascua era el mayor parche de basura en el Gran Pacífico, que, como se explicó anteriormente, fue creado por las corrientes oceánicas en las que los desechos se acumulan. También se conoce como el "séptimo continente" o como parte de la "sopa de plástico" (Charaf, 2015). Esta zona contaminada alrededor de la Isla de Pascua muestra los efectos desiguales de distribución de la movilidad de los plásticos causados por los flujos ambientales.

Los habitantes de la isla se ven especialmente afectados debido a la tremenda presión sobre los servicios y bienes causada por la cantidad de plástico que llega a sus costas o que es traída desde Chile por buques de carga y por turistas. Cada primer jueves de mes, un avión de carga se lleva dos toneladas de plástico para reciclar desde la isla hasta el continente (DW, 2016). Esto es mucho menos que la cantidad de desechos producida por los turistas en la isla todos los días, la cual se acerca a las siete toneladas. Podemos argumentar que, debido al área aislada, los turistas tienden a traer más productos plásticos del continente, por la suposición de la falta de disponibilidad de éstos en la isla. Giddens (1990, como se afirma en Ampuja, 2011) afirma que un desarrollo importante que se da junto con la globalización es "la intensificación de las relaciones sociales mundiales que vinculan localidades distantes de tal manera que los acontecimientos locales están formados por eventos que ocurren a muchas millas de distancia y viceversa versa "(p.283). El consumo de plástico en la Isla de Pascua, está determinado por las demandas del consumidor a muchos kilómetros de distancia, en un contexto social y geográfico muy diferente. Incluso si la isla no vendiera nada de plástico, debido al turismo y a las corrientes oceánicas, el plástico seguiría fluyendo hacia la isla. Además, lograr una prohibición de todos los plásticos es una tarea muy difícil, ya que la isla necesita importar alimentos y bebidas, artículos de higiene para hoteles y otros artículos envueltos en plástico. Estos flujos plásticos también están conectados con la dimensión social. Los isleños se ven directamente afectados por la cantidad de plástico, ya que el plástico es descartado en las zonas rurales, fuera de la vista de los turistas donde viven la mayoría de los isleños (Wood et al.2019). 
Dejando a un lado el comercio como causa de la globalización del plástico, también se destaca el aspecto social. En la Isla de Pascua, la mayoría de las personas trabajan como agricultores, pescadores, artesanos, en pequeñas empresas gubernamentales o en la industria del turismo. Desde que la industria del turismo comenzó a ser rentable, muchos chilenos se han mudado del continente a la Isla de Pascua para abrir hoteles y restaurantes. Esto, junto con el creciente número de turistas en comparación con la población local, ha causado una presión fuerte en el ambiente y en las infraestructuras. En 2002, el número de turistas era más o menos el mismo que el número de la población local. Sin embargo, donde el número de habitantes solo creció ligeramente (hasta 6658 habitantes en 2018), el número de turistas creció a más de 95.300 en 2018. Este flujo de personas y el establecimiento de negocios también conllevan ciertas necesidades materiales. Ha habido tensiones en torno a la cantidad de alimentos, agua potable, combustible y otros bienes materiales disponibles en la isla, ya que esta no puede abastecer a todos. Debido a estas tensiones, los habitantes originales a menudo se describen como "víctimas de la inmigración indiscriminada" (Long, 2014).

En términos de la sociología de flujos, podemos argumentar que las tensiones resultantes de no tener acceso a ciertos bienes son una forma de desigualdad. Los isleños son víctimas del turismo como causa de la globalización porque el acceso limitado a los bienes reduce su calidad de vida. Como lo enfatizan Mol y Spaargaren (2005), el "acceso a" no solo debe verse como el acceso directo a, por ejemplo, bienes materiales empacados en plástico, sino también la capacidad de influir en la velocidad, dirección e intensidad de los flujos de plástico. Cuando no se toman medidas apropiadas, la intensidad de los flujos de plástico conduce a mayores problemas ambientales y de salud. Njeru (2006) identifica múltiples problemas causados por los residuos plásticos, como la posibilidad de inundaciones causadas por bolsas de plástico que podrían bloquear los desagües, el consumo de plástico por parte del ganado que puede provocar su muerte, la disminución de fertilidad del suelo, los gases tóxicos liberados con su quema, y, por último, la creciente probabilidad de contraer malaria o dengue ya que las bolsas de plástico son hábitats de reproducción para los mosquitos.

Estos posibles problemas conllevan efectos importantes en la población originaria de la Isla de Pascua, ya que el ganado y la pesca son dos de sus actividades básicas. La población de peces se ve amenazada en número y calidad debido a la cantidad de microplásticos en el agua que rodea la isla, lo que también afecta al turismo. La posibilidad de malaria y los gases tóxicos de la quema de plásticos son amenazas directas a la salud tanto de locales como visitantes, ya que los residuos plásticos continúan llenando la isla. Esto podría conducir a una disminución del turismo y a una mayor probabilidad de problemas de salud, especialmente para la población local, perpetuando la injusticia de la desigual distribución global de los flujos de residuos plásticos.

Al analizar las consecuencias locales del plástico como residuo globalizado y sus impactos en la isla de Pascua, podemos argumentar que el bienestar de la isla y de sus habitantes está en riesgo. Las tensiones que surgen a partir de los efectos globalizadores del turismo parecen magnificar estas consecuencias negativas. Sin embargo, los isleños siguen siendo optimistas en la lucha contra el problema del plástico a través de diversas iniciativas locales. La isla recibió recientemente atención mundial sobre su problema plástico debido al estreno del documental "Comiéndose a Pascua", realizado por un director nacido en la Isla de Pascua (Rapu, 2019). A través de este documental, se buscaba crear conciencia sobre un problema global, que parece mucho más intenso y aparente al verse en pequeñas islas remotas. Su documental llamó la atención de la ONG internacional Plastic Oceans, cuyo objetivo es acabar con la contaminación plástica. Junto con empresas locales y otras organizaciones de conservación, ahora organizan limpiezas masivas de playas, talleres educativos e 
investigaciones no solo para inspirar, sino también para hacer un cambio. También organizaron la campaña "Nadar en contra del plástico" en marzo de 2019, en la que una nadadora de resistencia que batió récords está nadando durante 63 horas por 63 kilómetros alrededor de la isla en busca de donaciones para combatir la contaminación plástica (Hardin, 2019).

Estos ejemplos son apenas un inicio de un movimiento de resistencia local que puede ir tomando más forma a medida en que los locales se organicen internamente y puedan compartir su historia con el resto del mundo. De esta manera los locales también pueden utilizar los efectos globalizadores del turismo como una herramienta que les dé una posibilidad de combate en la intensa batalla contra la contaminación plástica.

\section{CONCLUSIÓN}

Este artículo examinó la naturaleza de los flujos globales de movilidad plástica y cómo se intensifican por los efectos globalizadores de la industria del turismo. Al considerar el plástico como un flujo material, se pudo resaltar los principales impulsores y problemas en los procesos globales de producción, consumo y posconsumo de plástico, así como las relaciones de poder, en las que algunos actores tienen el control sobre la movilidad y los flujos de los plásticos. Los diferentes impulsores tienen una correlación directa con los factores económicos y sociales ejemplificados por los hábitos de producción y consumo. China y Europa son los mayores productores de plásticos (Ritchie \& Roser, 2018; PlasticsEurope, 2017), esto les da el poder para gobernar los flujos de materiales, especialmente cuando están relacionados con el comercio.

En el proceso de reciclaje global, su capacidad de procesamiento de plásticos, le dio a China la capacidad de afectar localidades distantes al imponer una prohibición que limitaba y direccionaba el flujo de residuos plásticos hacia otros lugares. Por otro lado, los plásticos que terminan en el océano son una gran preocupación ambiental y dado que estos plásticos a menudo se dirigen hacia parches de basura con ayuda del viento y de las corrientes, se convierten en preocupaciones aún mayores para las islas remotas cercanas a estos parches. Estos flujos de plásticos impulsados por corrientes naturales no controladas por el hombre, no solo dañan la fauna marina, sino también el medio ambiente en la isla y la vida de sus habitantes. Además de las corrientes oceánicas y el viento, el turismo también contribuye al aumento de la contaminación plástica en el océano (Ivar do Sul et al., 2011).

El turismo está interrelacionado con varios impulsores de los flujos de plástico, principalmente al alterar los patrones de producción y consumo a través de influencias directas e indirectas. Se considera que las influencias directas están interrelacionadas principalmente con el proceso de consumo, que se encuentra al final de la cadena de valor del turismo. Las influencias indirectas, por lo tanto, explican todas las actividades que deben realizarse para satisfacer las necesidades de los turistas antes de esta etapa final y que afectan los flujos del plástico.

El turismo no solo afecta a los residuos plásticos, sino que también se ve directamente afectado por ellos, como se ilustra en el caso de la Isla de Pascua. La capacidad de generar alimentos en las islas remotas se ven afectadas por la contaminación plástica con la ingesta de plástico en peces y ganado. El plástico también disminuye la calidad de vida de sus habitantes, ya que los residuos plásticos se eliminan en las zonas rurales donde viven la mayoría de los isleños y se ejerce una enorme presión sobre los servicios y bienes. Esto 
podría afectar directamente al turismo, ya que es probable que surjan conflictos una vez que los habitantes locales se cansen de las relaciones desiguales generadas por el turismo y la globalización, en las que se encuentran en el extremo "perdedor". Los conflictos pueden llevar a los locales a rechazar un mayor desarrollo del turismo, perder la confianza en las instituciones reguladoras o simplemente deteriorar su relación con los turistas.

La acumulación de plásticos en islas remotas tiene consecuencias ambientales que disminuyen la calidad de los recursos naturales. La fauna marina, uno de los mayores atractivos de los destinos costeros, se ve directamente perjudicada por la presencia de microplásticos y macroplásticos en el océano. Si el número o la calidad de los recursos marinos disminuyen demasiado, los turistas probablemente buscarán otra isla para el buceo recreativo. Por lo tanto, un entorno deteriorado podría causar una disminución en el número de turistas.

La Isla de Pascua es, como muchas otras islas remotas, víctima de procesos globales. Sin embargo, la visibilidad que la isla ha ganado a través del turismo a nivel global, también ha sido utilizada por los locales para combatir estos procesos. La localidad ha utilizado estos canales globalizadores de manera inversa, enviando un mensaje de la localidad al mundo. Las iniciativas locales como talleres educativos, limpieza de playas y campañas ayudan a desacelerar los efectos negativos de los residuos plásticos globales. Aunque las fuerzas de movilización siguen sobrepasando en gran manera a los impulsos locales, los esfuerzos de la localidad recién comienzan y los efectos positivos alcanzados hasta el momento pueden impulsar un continuo interés de las autoridades responsables a distintos niveles, dando así una esperanza para el bienestar de la isla y sus habitantes.

\section{AGRADECIMIENTOS}

Le agradecemos al Dr. Rene van der Duim de la Universidad de Wageningen por los comentarios y apoyo dados para la realización de este artículo.

\section{REFERENCIAS}

5 Gyres, Clean Production Action, Surfrider Foundation, UPSTREAM (2016). The Plastic Ban List [La Lista de la Prohibición del Plástico], Accesado Mayo 9 2019, desde: https://d3583ivmhhw2le.cloudfront.net/images/uploads/publications/PlasticsBANList 2016.pdf

About Plastics Packaging. (n.d.). Accesado Mayo 9, 2019 desde: https://www.bpf.co.uk/plastipedia/applications/about_plastics_packaging.aspx

ABTA (2019) Make holidays greener [Por unas vacaciones más verdes]. Accesado, Mayo 1 2019, desde: https://makeholidaysgreener.com/holidaymaker

American Chemistry Council (2019). Life cycle of a plastic product [El ciclo de vida de un producto plástico]. Accesado Abril 22 2019, desde: https://plastics.americanchemistry.com/Lifecycle-of-a-Plastic-Product/

AL-RODHAN, N. R., \& STOUDMANN, G. (2006). Definitions of globalization: A comprehensive overview and a proposed definition [Definiciones de globalización: Una revisión amplia y una nueva propuesta de definición]. Program on the Geopolitical Implications of Globalization and Transnational Security, 6(1-21). 
AMPUJA, M. (2012). Globalization theory, media-centrism and neoliberalism: A critique of recent intellectual trends [Teoría de globalización, media centrismo y neoliberalismo: Una crítica de las recientes tendencias intelectuales]. Critical Sociology, 38(2), 281301.

BLICHERT, F. (2019, March 8). Exclusive Trailer: "Eating Up Easter" to head to PBS following Easter Island Premiere [Trailer Exclusivo: "Devorando Pascua"]. Accesado Marzo 24, 2019, desde: http://realscreen.com/2019/03/08/exclusive-trailer-eating-upeaster-to-head-to-pbs-following-easter-island-premiere/

CHARAF, P. (2015). "Disenchanted": Easter Island - A Paradise Devastated by Plastic Pollution [Desencantado: Isla de Pascua - Un paraíso devastado por el plástico]. National Geographic.

DINDA, S. (2006). Globalization and Environment: Can Pollution Haven Hypothesis alone explain the impact of Globalization on Environment? [Globalización y medio ambiente: ¿Puede la hipótesis del refugio de la contaminación explicar por sí sola el impacto de la globalización en el medio ambiente?].

DIVISEKERA, S. (2010). Economics of tourist's consumption behaviour: Some evidence from Australia [Economía del consumo del turista: evidencia de Australia]. Tourism Management, 31(5), 629-636. doi:10.1016/j.tourman.2009.07.001

DW (2016, Septiembre 14) Easter Island, the tourists and the garbage [Isla de Pascua, los turistas y la basura]. Article Accesado Marzo 24, 2019, desde: https://www.dw.com/cda/en/easter-island-the-tourists-and-the-garbage/a-19549563

Easter Island Tourism (2019). Scuba Diving Accesado Mayo 4, 2019, desde: http://www.easterislandtourism.com/turistic-info/scuba-diving/

ESCOBAR, A. (1998). Whose Knowledge, Whose Nature? Biodiversity, Conservation, and the Political Ecology of Social Movements [¿De quién es el conocimiento, de quién es la naturaleza? Biodiversidad, conservación y ecología política de los movimientos sociales]. Journal of Political Ecology (p. 53 - 82)

FABINYI, M. (2010). The intensification of fishing and the rise of tourism: competing coastal livelihoods in the Calamianes Islands, Philippines [La intensificación de la pesca y el despertar del turismo: medios de vida en competencia en las Islas Calamianes, Filipinas]. Human Ecology, 38(3), 415-427.

GABBATISS, J. (2018, June 7). Tourists visiting the Mediterranean this summer cause 40 per cent spike in plastic, report reveals [Los turistas que visitan el Mediterráneo este verano causan un aumento del $40 \%$ en el plástico]. Accesado 2019, April 5, desde: https://www.independent.co.uk/environment/plastic-pollution-tourists-mediterraneansea-increase-microplastics-wwf-a8388296.html

GAIA, Global Alliance for Incinerator Alternatives (2019). Discarded: Communities on the frontlines of the global plastic crisis [Descartados: Comunidades al frente de la crisis plástica global]. Accesado desde: https://wastetradestories.org/wp-content/uploads/ 2019/04/Discarded-Report-April-22.pdf

GROSS, M. (2017). Our Planet Wrapped in Plastic [Nuestro planeta envuelto en plástico]. Current Biology Magazine(p.785 - 788)

HAMES, R. (2007). The Ecologically Noble Savage Debate [El debate del salvaje ecológicamente noble]. Annual Review of Anthropology. Vol. 36, (p.177-190)

HARDIN, T. (2019). Swim against plastic: Easter Island [Nadar contra el plástico: Isla de Pascua]. Plastic Oceans. 
HESKETT, M., TAKADA, H., YAMASHITA, R., YUYAMA, M., ITO, M., GEOK, Y. B., \& Powell, T. (2012). Measurement of persistent organic pollutants (POPs) in plastic resin pellets from remote islands: Toward establishment of background concentrations for International Pellet Watch [Medición de contaminantes orgánicos persistentes (COP) en gránulos de resina plástica de islas remotas: hacia el establecimiento de concentraciones de fondo para International Pellet Watch]. Marine Pollution Bulletin, 64(2), 445-448.

Hospitality Insights (2019). Single-use plastics: Hotels and airlines are cutting down [Plásticos de un solo uso: los hoteles y las líneas aéreas están reduciendo]. Accesado Mayo 1, 2019 desde: https://hospitalityinsights.ehl.edu/hotels-single-use-plastics-ban

IVAR DO SUL, J. A., SANTOS, I. R., FRIEDRICH, A. C., MATTHIENSEN, A., \& FILLMANN, G. (2011). Plastic Pollution at a Sea Turtle Conservation Area in NE Brazil: Contrasting Developed and Undeveloped Beaches [Contaminación plástica en un área de conservación de tortugas marinas en el noreste de Brasil: contrastando playas desarrolladas y subdesarrolladas]. Estuaries and Coasts, 34(4), 814-823.

LAVILLE, S., \& TAYLOR, M. (2018, January 17). Nearly 1m tonnes every year: supermarkets shamed for plastic packaging [Casi 1 millón de toneladas cada año: supermercados avergonzados por los envases de plástico]. Accesado 2019, April 24, desde: https://www.theguardian.com/environment/2018/jan/17/nearly-1m-tonnesevery-year-supermarkets-shamed-for-plastic-packaging

LONG, G. (2014). Trouble in Paradise for Chile's Easter Island [Problemas en el paraíso para la Isla de Pascua en Chile]. BBC News.

MOL, A. P., \& SPAARGAREN, G. (2005). From additions and withdrawals to environmental flows: reframing debates in the environmental social sciences [De adiciones y retiros a flujos ambientales: reformulando debates en las ciencias sociales ambientales]. Organization \& Environment, 18(1), 91-1

MONTEIRO, R. C., IVAR DO SUL, J. A., \& Costa, M. F. (2018). Plastic pollution in islands of the Atlantic Ocean [Contaminación plástica en las Islas del Atlántico]. Environmental Pollution, 238, 103-110. doi:10.1016/j.envpol.2018.01.096

MUNAR, A. M. (2007). Rethinking globalization theory in tourism [Repensando la teoría de la globalización en turismo]. Tourism Culture \& Communication, 7(2), 99-115.

NJERU, J. (2006). The urban political ecology of plastic bag waste problem in Nairobi, Kenya [Ecologías políticas del problema de las bolsas de plástico en Nairobi]. Geoforum 37, p. 1046 - 1058.

PlasticsEurope (2017). Plastics - the Facts 2017, an analysis of European plastics production, demand and waste data [Plásticos - los hechos 2017, un análisis de la información sobre la producción del plástico, demanda y desecho].

PRICE, J. F., WELLER, R. A., \& SCHUDLICH, R. R. (1987). Wind-driven ocean currents and Ekman transport [Corrientes oceánicas causadas por el viento y el transporte Ekman]. Science, 238(4833), 1534-1538.

RAPU, S. (2019). Eating up Easter [Comiéndose a Pascua]. Accesado Marzo 20, 2019 desde: https://eatingupeaster.com

RITCHIE, J. B., \& ZINS, M. (1978). Culture as determinant of the attractiveness of a tourism region [La cultura como determinante del atractivo de una region turística]. Annals of Tourism Research, 5(2), 252-267

RITCHIE, H. \& ROSER, M. (2018). Plastic Pollution. Our World in Data [Contaminación Plástica, nuestro mundo en datos]. Accesado 2019, March 29, from: https://ourworldindata.org/plastic-pollution

SEBILLE, E. (2015). The oceans' accumulating plastic garbage [Los océanos acumulando residuos plásticos]. Physics Today, 68(2), 60-61. doi:10.1063/pt.3.2697 
SHEAVLY, S. B., \& REGISTER, K. M. (2007). Marine debris \& plastics: environmental concerns, sources, impacts and solutions [Escombros marinos y plásticos: preocupaciones ambientales, fuentes, impactos y soluciones]. Journal of Polymers and the Environment, 15(4), 301-305.

SHELLER, M. \& URRY, J. (2006). The new mobilities paradigm [El nuevo paradigma de movilidad]. Environment and Planning, Vol. 38, p. 207 - 226

TURNER, D. A., WILLIAMS, I. D., \& KEMP, S. (2015). Combined material flow analysis and life cycle assessment as a support tool for solid waste management decision making [Análisis combinado de flujo de material y evaluación del ciclo de vida como herramienta de soporte para la toma de desiciones del manejo de resíduos sólidos]. Journal of cleaner production, 129, 234-248.

WAHAB, S., \& COOPER, C. (2001). Tourism in the Age of Globalisation [Turismo en la era de la Globalización]. Routledge.

WILLIAMS, A. M., \& HALL, C. M. (2010). Tourism and migration: New relationships between production and consumption [Turismo y migración: Nuevas relaciones entre producción y consumo]. Tourism Geographies, 2(1), 5-27. doi:10.1080/146166800363420

WOOD, M. E., MILSTEIN, M. \& AHAMED-BROADHURST, K. (2019). Destinations at Risk: The Invisible Burden of Tourism [Destinos en Riesgo: La carga invisible del turismo] p. 2 - 56

ZOETE, T. (2018). De Chinese Afvalcrisis [La crisis China de Resíduos]. Recycling Netwerk. Accesado May 1, 2019 desde: https://recyclingnetwerk.org/2018/11/01/dechinese-afvalcrisis/ 